\title{
1 Ground subsidence monitoring with SAR interferometry techniques in \\ 2 the rural area of Al Wagan, UAE
}

3 Nikolaos Liosis $^{1 *}$, Prashanth Reddy Marpu ${ }^{1}$, Kosmas Pavlopoulos $^{2}$ and Taha

$4 \quad$ Ouarda $^{3}$

$5 \quad{ }^{1}$ Institute Center for Water and Environment, Khalifa University of Science and Technology,

6 Masdar Institute, Masdar City, P.O. Box 54224, Abu Dhabi, United Arab Emirates;

$7 \quad{ }^{2}$ Department of Geography and Planning, Paris-Sorbonne University Abu Dhabi, P.O. Box

838044 AD UAE; ${ }^{3}$ Laboratoires d'Hydraulique Institut National de la Recherche Scientifique

9 Centre Eau Terre Environnement 490, rue de la Couronne Québec (Québec) G1K9A9

10 Canada;

$11 *$ Corresponding author. Email: nliosis@masdar.ac.ae

\begin{abstract}
In this work, we investigate the past and present land deformation in Al Wagan area in the United Arab Emirates. The area is primarily an agricultural region where dependence on groundwater is documented. Such a reliance on ground water resources in a region which is characterized by very low precipitation can lead to significant land subsidence as was observed in this study which identified fast and localized deformation trends. The quantification of terrain distortions of large magnitude and small amplitude in this area with SAR Interferometry is a challenging task using moderate resolution data due to the incoherent surface background. Even though SAR acquisitions were sparse
\end{abstract}


over this region, the available ENVISAT, ALOS and Sentinel-1A imagery was analysed with differential interferometry and the Small Baseline Subset technique in order to provide fair estimates about the evolution of the deformation pattern in a limited area. The depletion of the aquifer resources which is confirmed from groundwater level data is speculated to be the most probable cause. However these assumptions require further investigation in order to discover a remediation for this problem.

Keywords: SAR Interferometry; SBAS; subsidence; groundwater; UAE

\section{Introduction}

Excessive groundwater extraction from the subsurface may lead to the depletion of the aquifer's natural resources, which potentially results in ground surface subsidence phenomena. The agriculture sector of the United Arab Emirates (UAE) has been expanding since the 1990s (Statistics Centre of Abu Dhabi, 2014), but since all these farms are situated in an arid region with a hot desert climate and extremely sparse precipitation events, they mostly relied on groundwater for their viability (Environmental Agency of Abu Dhabi, 2009). The direct implications of this are the diminishing natural aquifer resources of the UAE, which has already been analysed in another study (Gonzalez et al., 2016), the degradation of the water quality (Vrba, 2003; Al-Naeem, 2014) and the occurrence of localized terrain subsidence (Holzer and Galloway, 2005).

2 Differential Synthetic Aperture Radar (SAR) Interferometry (DInSAR) is one of the most effective remote sensing methods to quantify terrain distortions related to various 
44 causes over a large spatial scale (Bürgmann et al., 2000; Ferretti, 2014). The multi-temporal 45 stacking techniques that have been developed in the past two decades, like Persistent 46 Scatterers (PS) (Ferretti et al., 2001; Hooper et al., 2004) and Small Baseline Subset (SBAS)

47 (Berardino et al., 2002; Lanari et al., 2004), are capable of providing accurate estimations of 48 the displacement time series and the deformation velocities, with the latter method being 49 more suitable for rural regions as it is based on identifying Distributed Scatterers (DS) that 50 are more common in natural environments in a stack of small baseline interferograms. There 51 already exist numerous studies where groundwater related subsidence phenomena were 52 successfully assessed with SBAS techniques (Chaussard et al., 2014; Kim et al., 2015; 53 Artesea et al., 2016). However, the accuracy and the reliability of the results is affected by 54 temporal decorrelation in rural areas where incoherent surface types, like sand and vegetation 55 are the dominant land covers (Bamler and Hartl, 1998; Massonnet and Feigl, 1998). DInSAR applications are very sparse in the UAE mainly due to the extended presence 57 of the sandy geological background which significantly degrades the performance of the 58 process and the reliability of the final outputs. A previous study had demonstrated the 59 application of SBAS over an extended area of the Eastern part of the UAE focusing on the 60 most coherent parts and providing rough indications about the locations of particular ground 61 distortion zones (Cantone et al., 2013). However, to the authors' knowledge, there are no 62 existing studies attempting to monitor localized surface deformations in the UAE at small 63 scales. The major challenge in this rural area where the subsidence phenomena were 64 extremely fast and localized was to define the optimum data resolution that allowed the 65 detection and depiction of the real deformation magnitude in the presence of high 66 decorrelation noise. 

regions affected by subsidence phenomena induced by subsurface resources exploitation and

69 to provide quantitative estimations about the spatiotemporal patterns of the terrain 70 displacements. The objective of the present work is to analyse the observations obtained by

71 conventional DInSAR and SBAS techniques and to demonstrate that despite the presence of

72 low coherence that obstructs the interferometric processing, reliable results may still be 73 obtained to provide a comprehensive overview of the subsidence phenomenon in the study 74 area over a larger spatial extent than is possible by sparse permanent GPS stations.

75

\section{Study Area}

Al Wagan is located on the eastern part of the Abu Dhabi Emirate, approximately 65 kilometres southwest of the city of Al Ain at the borders with Oman. Figure 1a depicts the study area along with the locations of monitored wells from the Environmental Agency of Abu Dhabi (EAD). It is a hot and arid region at the edge of the Rub' al Khali desert with a generally flat terrain - except for the star dunes - at an average altitude of 170 meters. The surrounding area of this sparsely populated town facilitates many farms at the west side of the borders which unequally spread up to 18 kilometres towards the desert gradually fading out. The farms along with other vegetated areas cover around $71 \mathrm{~km}^{2}$ over a total area of approximately $357 \mathrm{~km}^{2}$.

The borders of the UAE with Oman at the study area roughly depict the transition from the fluvial deposits in the east to the Aeolian deposits to the west. The geological background of the north part of the region where the main settlement is sited consists of fluvial quaternary deposits which are composed of a mixture of sandstone, conglomerates 
90 and siltstone (Hili formation), formed by torrent activity originating from the Omani

91 mountains. The central and southern parts are mainly covered by low and star dunes of sandy

92 material transferred by aeolian processes. The heterogeneity of the local geology shown in

93 figure $1 \mathrm{~b}$ is clearly visible in radar and optical satellite imagery and sporadic shifts between

94 these two main background types are not uncommon. The geological map of the area is not

95 totally reflected on the land use map (figure 1c), implying that the farms are not located

96 exclusively on the alluvial fans but on the sand deposits as well.

97 There were no active faults in the study area observed in the geological maps neither

98 mentioned in literature. The nearest regions that were affected by active tectonism in the

99 studied period were the Fujairah Mountains and the Musandam Peninsula (Yagoub, 2015).

100 Therefore it was considered in this study that the effects of earthquakes on surface

101 deformations were negligible and they were not taken into account. 

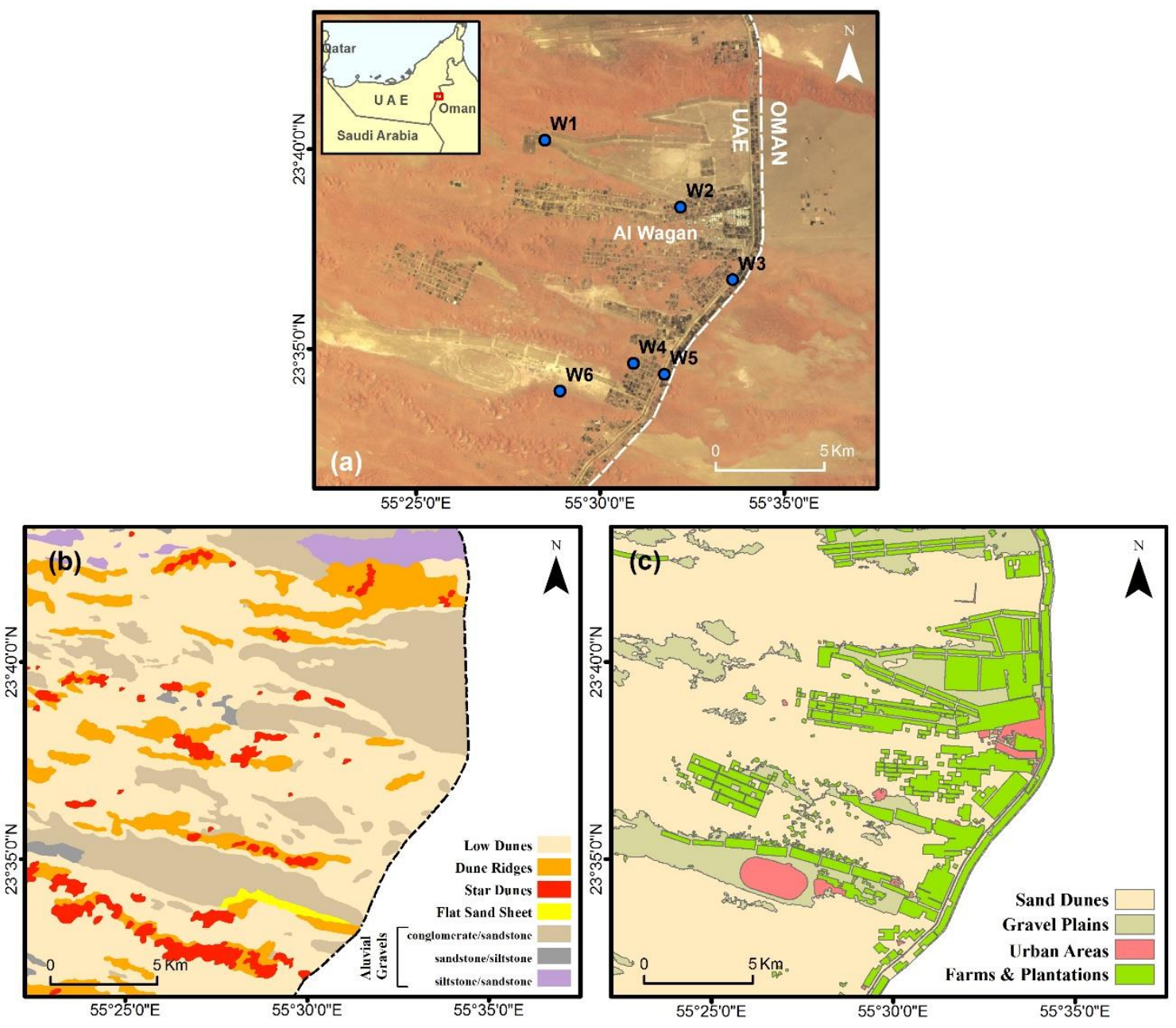

104 Figure 1. a) Landsat 8 composite image (bands 4, 3, 2) of the study area dated 26/5/2017. b) 105 Geological map (BGS) c) Generalized land cover map (EAD).

\section{Data}

The interferometric analysis was performed with the use of the freely available C-

109 band and L-band SAR data covering the study area. Two C-band image stacks acquired from the ENVISAT satellite by the Advanced Synthetic Aperture Radar (ASAR) sensor were

111 obtained from the European Space Agency (ESA). The descending stack consists of 32 112 acquisitions covering the period 7/8/2003 - 22/4/2010, while the ascending includes only 10 
113 images from the period 3/4/2007 - 14/10/2008. Other C-band data used in this study were

114 the available Sentinel-1A acquisitions over the area of interest, but the sparsity of the data

115 did not allow a proper time series analysis. Three SAR acquisitions were used for differential

116 interferogram generation dated 18/2/2016, 2/12/2016 and 8/3/2017. The L-band data were

117 also sparse over this region, consisting of 7 ALOS PALSAR acquisitions from the period

118 5/8/2007 - 10/8/2009 obtained from the Alaska Satellite Facility (ASF). The general

119 characteristics of these radar data are presented in table 1.

120

121

Table 1. Main characteristics of the SAR data used in the study.

\begin{tabular}{|c|c|c|c|c|c|}
\hline Sensor & $\begin{array}{c}\text { Number of } \\
\text { images }\end{array}$ & Pass & Track/Frame & $\begin{array}{c}\text { Acquisition } \\
\text { Mode }\end{array}$ & Polarization \\
\hline ENVISAT/ASAR & 32 & Descending & $435 / 3123$ & IW-IM & VV \\
\hline ENVISAT/ASAR & 10 & Ascending & $13 / 459$ & IW-IM & VV \\
\hline ALOS/PALSAR & 4 & Ascending & $460 / 567$ & FBD & HH-HV \\
\hline ALOS/PALSAR & 3 & Ascending & $460 / 567$ & FBS & HH \\
\hline Sentinel-1A & 3 & Ascending & 130 & IW & VV-VH \\
\hline
\end{tabular}

122

The topographic phase removal was performed with the 3 arcsecond Digital Elevation

124 Model (DEM) of the Shuttle Radar Topography Mission (SRTM). Other data that were used

125 include the geological map of the area produced by the British Geological Survey (BGS)

126 under contract from the Ministry of Energy of the UAE, the Terrestrial Habitat map of the

127 UAE and the groundwater levels from the monitored wells of the study area obtained from

128 the Environmental Agency of Abu Dhabi (EAD) in the period 5/3/2000 - 9/9/2015. 


\section{Methods}

DInSAR relies on the isolation of the deformation phase component which corresponds to the terrain movements between the two SAR acquisitions of similar geometry. The Single Look Complex (SLC) images of each stack were coregistered on a single master scene in order to compute the interferograms. Due to the flat relief of the study area the SRTM DEM of $90 \mathrm{~m}$. resolution was considered adequate for subtraction of the local topography, since differences between trials with the ASTER and the $30 \mathrm{~m}$. resolution SRTM DEMs were insignificant. The Goldstein method was used to apply frequency domain phase filtering to the differential interferograms (Goldstein and Werner, 1998) with small windows in order to avoid aliasing fast deformation fringes, at the expense of preserving a lot of decorrelation noise in the final outputs. After the phase unwrapping stage (Pepe and Lanari, 2006), orbital refinement was performed for estimation and removal of residual phase ramps from the unwrapped interferometric stack by selecting ground control points common for all the generated interferograms.

The SBAS technique is based on generating and stacking a series of interferograms with small perpendicular baselines in order to reduce the spatial decorrelation effects. Therefore, the DInSAR process was applied to interferograms that satisfied certain temporal/perpendicular orbit criteria depending on the monitored surface types, the critical baseline and the relative space-time dispersion of the acquisitions in each stack. At the first inversion of the SBAS algorithm a primary estimate of the mean velocity field was obtained by applying a low degree displacement model to the unwrapped phases. This low pass deformation component along with possible topographic remnants was subtracted from the 
152 wrapped interferograms. Subsequently, the residual phase was unwrapped and added back to

153 the subtracted deformation signals (Berardino et al., 2002).

In the second step the refined unwrapped interferograms were inverted with a

155 Singular Value Decomposition (SVD) approach in order to derive the time series of the

156 displacements (Lanari et al., 2007). The atmospheric signals were removed with low pass

157 spatial and high pass temporal filtering operations (Ferretti et al., 2000) and the final

158 deformation history and velocity of the multi-temporally coherent pixels was extracted along

159 with the topographic residuals between the DEM and the actual ground surface. The results

160 were geocoded in UTM zone 40N projected coordinate system and the data processing was

161 mainly performed with Sarscape software. The general workflow of the interferometric

162 processes applied in this study is summarized in figure 2.

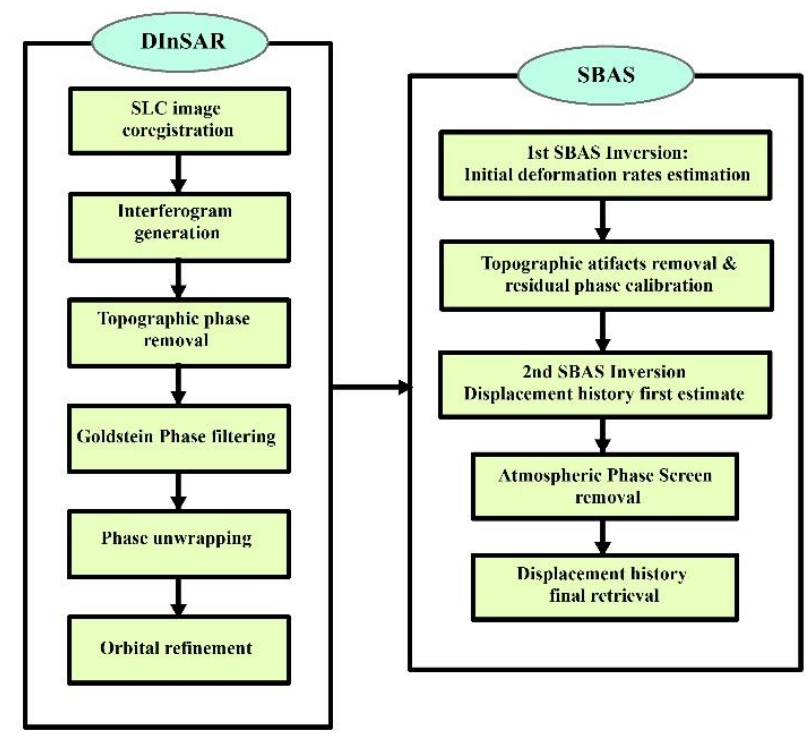




\section{Interferometric processing and results}

169

171

172

173

174

175

176 177 days.

178

\section{ENVISAT data}

Since the acquisitions of the descending stack were temporally irregular with a gap period from July 2004 to February 2006 (figure 4b), the thresholds for interferogram generation were set to 800 days temporal / $360 \mathrm{~m}$. perpendicular baseline in order to avoid underlaps in the stack. The coherence maps showed that the alluvial gravels were remaining generally coherent for more than two years in short baseline interferometric pairs, but the coherence of the sand dune areas was completely lost within 35 days. The block structured farms located in the sandy areas maintained their coherence at moderate levels for 100 - 150

Despite the low phase signal quality the differential interferograms revealed a fast subsidence pattern extended throughout the whole farmland area with the deformation magnitude along the Line-Of-Sight (LOS) peaking at several points within the region, especially along the west side of the highway that links the border settlements. In the central and south regions, the deformations were even greater. But this observation relied only on small temporally separated pairs as temporal decorrelation prevented from obtaining long term estimations. A seasonal variation was also observed as the summer period pairs tended to exhibit larger displacement signals than those of the winter season, reaching almost one fringe per month south of the town of Al Wagan. If the observed subsidence was related to groundwater pumping, the increased water demands of agriculture in a desert environment during the hot and dry periods could explain this phenomenon. These primary observations were consistent in all the temporally coherent interferograms of both the ascending and the descending stacks, which reinforced the initial assumption of a fast subsidence phenomenon. 

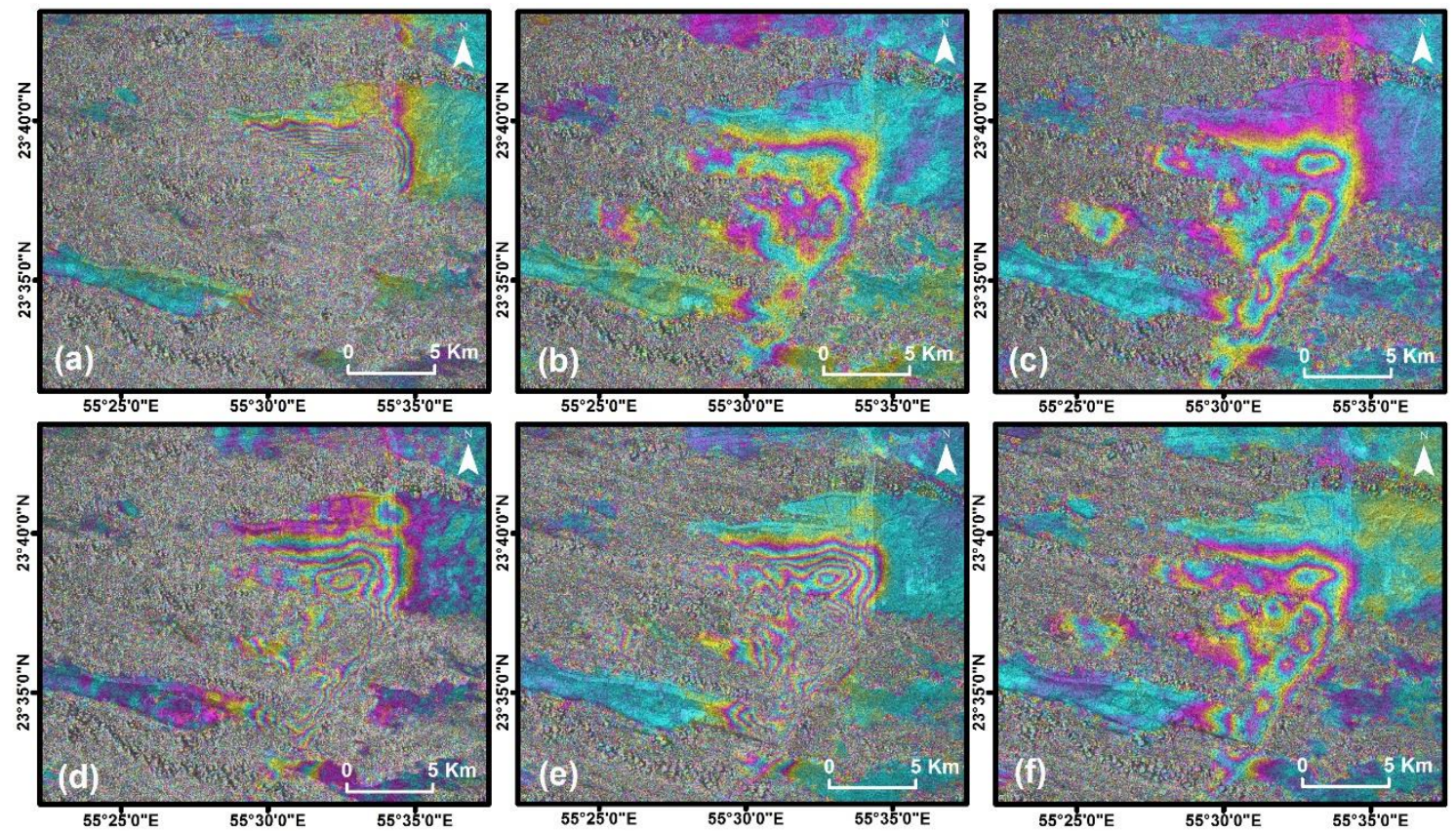

194 Figure 3. Differential interferograms from ENVISAT pairs. a) From descending pair 3/4/2004 195 9/3/2006, where temporal and fast fringes decorrelation are dominant in long time spans. b) 196 Descending pair 29/11/2007 - 17/4/2008, indicative of the lower fringe rates in the winter seasons. c) 197 Descending pair of 17/4/2008 - 26/6/2008. d) Descending pair 7/5/2009 - 11/2/2010, where the 198 deformation pattern is clear despite the decorrelation noise. e) Ascending pair 3/4/2007 - 12/2/2008. 199 f) Ascending pair 8/1/2008 - 27/5/2008.

202 deformation gradient, which theoretically is one fringe per pixel (Massonnet and Feigl, 203 1998). Since in reality this threshold is significantly lower due to the presence of 204 decorrelation noise in low coherent areas (Baran et al., 2005), and fast fringes decorrelation 205 was observed in all the interferograms of more than two years separation, the images were 
multilooked 1 x 5 (range $\mathrm{x}$ azimuth). For larger factors, the deformation pattern became undetectable or ambiguous even in temporally adjacent pairs.

In order to obtain an initial estimation of the deformation velocities, interferometric stacking was applied (Strozzi et al., 2001) after the phase unwrapping stage with pairs of short perpendicular baselines $(<250 \mathrm{~m})$. Due to temporal decorrelation and phase unwrapping failures the reliability of the output linear deformation velocities was compromised, however the areas of stability were delineated for subsequent reference point selections.

The interferograms of the descending stack were then used as inputs for the SBAS algorithm. After discarding the pairs with significant unwrapping errors, 84 interferograms were inverted in order to extract the displacement history and velocities of the multitemporally coherent points. The relative time-baseline position of the images is shown in figure $4 \mathrm{~b}$. The resulting deformation maps showed that cumulative subsidence exceeded 1 meter and LOS displacement rates reached $-18 \mathrm{~cm} /$ year in the most affected areas during the sampling period, with the time series exhibiting a small seasonal effect. However, the coherent pixel grid was sparse at the central and south parts of the study area where subsidence appeared larger in the short temporal baseline interferograms, exhibiting displacements equal or lower than those of the north part. Decorrelation and unwrapping errors prevented from obtaining accurate estimates in those regions.

Figure 4a depicts the vertical deformation rates in the study area (valid under the assumption of negligible horizontal component). Point P1 is located in the northernmost part of the region where even though displacement rates were low, an accelerating subsidence trend was observed after 2007. P2 is sited in the fast subsiding area near the town at the local 
229 deformation maximum, while $\mathrm{W} 2$ is a monitored well within the same area. P3 and P4 are 230 representative points of the central and south subsiding regions respectively. Even though

231 according to the displacement history of figure 4c they exhibit lower or equal deformations 232 with point $\mathrm{P} 2$, it is noted that coherence levels were marginal in these areas and a sufficient 233 density of measure points was not achieved.

234 As a general observation, the linear model that was applied to the displacement 235 velocities could roughly capture the seasonal behaviour of the ground deformations. It is also 236 noted that a quadratic model was a more appropriate fit in the southern region proximate to $237 \mathrm{P} 4$ as it was in the area of $\mathrm{P} 1$, with the subsidence phenomenon slightly accelerating after 238 2006. In either case, it is not certain whether this was due to the higher frequency of 239 acquisitions after that year (figure $4 b$ ), since the large temporally separated pairs of the 240 preceding gap period included many phase inconsistencies in the low coherent areas.

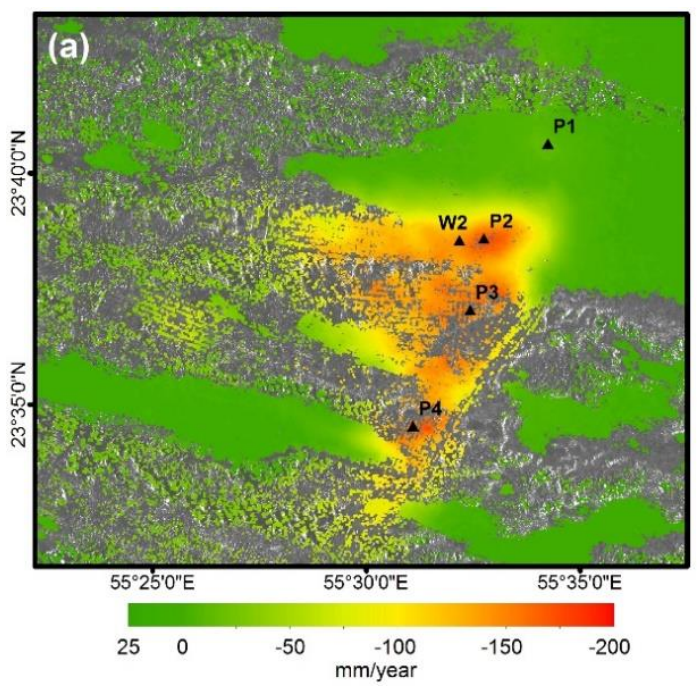




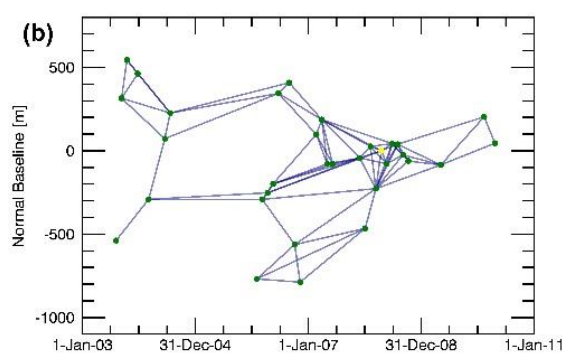

245

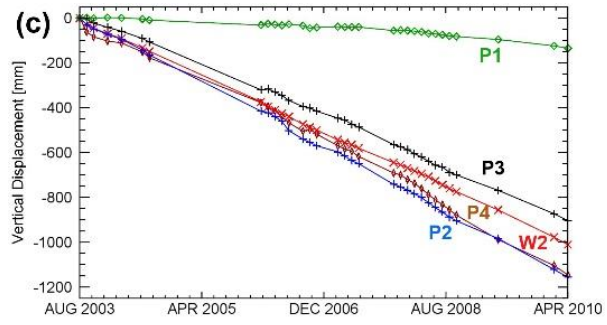

248 Figure 4. a) Vertical displacement velocities of the period 2003 - 2010. b) Time position plot 249 of the descending stack images, where nodes and arcs represent scenes and interferograms 250 respectively. c) Displacement history of selected points in the study area.
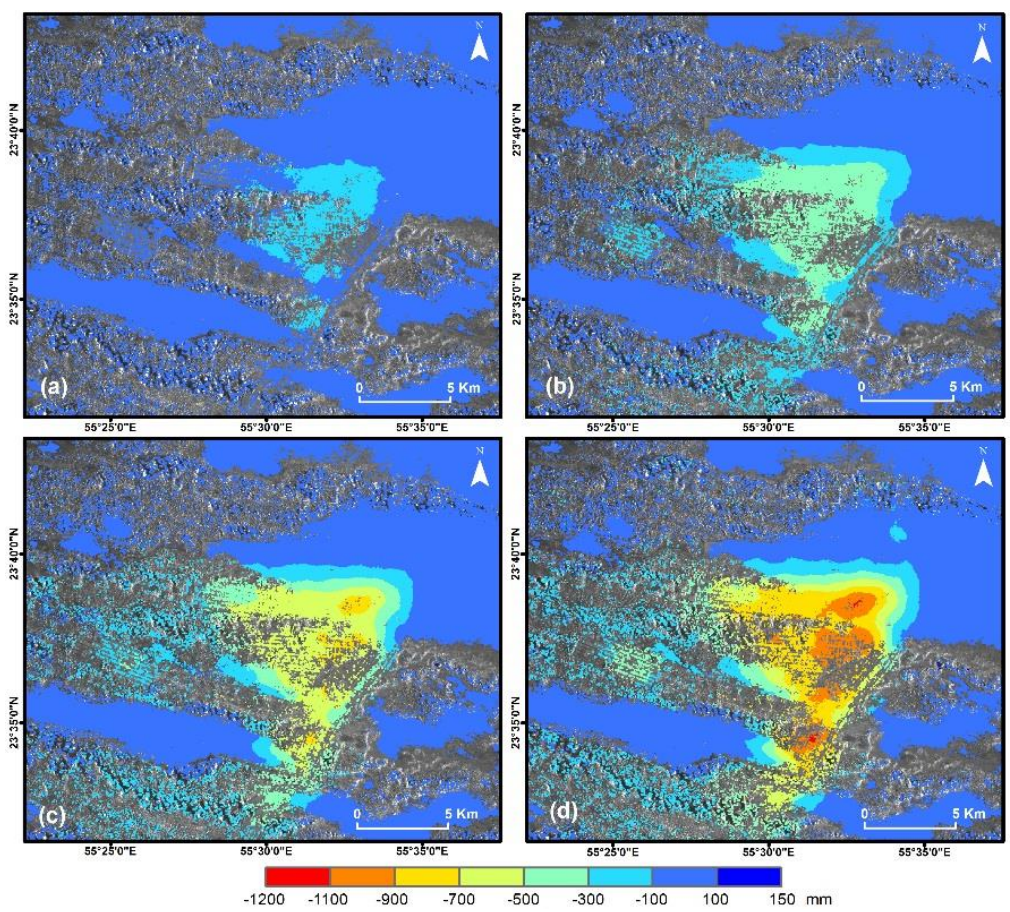
254 Figure 5. Evolution of the LOS deformation pattern obtained from the descending 255 acquisitions. a) 7/8/2003 - 22/7/2004. b) 7/8/2003 - 22/6/2006. c) 7/8/2003 - 22/5/2008. d) $2567 / 8 / 2003-22 / 4 / 2010$. within 400 days temporal separation and $330 \mathrm{~m}$. maximum normal baseline for comparison with the results obtained from the descending stack. The generated maps replicated the ground distortion pattern of the previous implementation which verified that the vertical movements were dominant, considering the similar incident angle of the ascending and 263 descending acquisitions. The cumulative LOS displacements derived from the two stacks 264 were comparable in the northern area during similar observation periods. However, in the central and southern regions of the study area, where the multitemporal coherence was increased in the ascending stack, the deformations of the 267 commonly coherent pixels appeared significantly larger than those estimated from the 268 descending stack. These local deviations were mostly attributed to phase inconsistencies 269 induced by decorrelation noise in the interferograms of the descending stack rather than to a 270 horizontal component of the terrain movements. The coherence maps of both stacks are 271 shown in figure 6.

272 The comparison of the cumulative LOS displacements between the two stacks in the 273 sampling period of the ascending acquisitions is demonstrated in figures $7 \mathrm{a}$ and $\mathrm{b}$. Figure $7 \mathrm{~d}$ 274 illustrates the vertical displacement history of the selected points in the study area after the 275 application of a linear model to the subsidence rates. Points P1, P2 and W2 follow the same 
276 pattern as derived from the descending stack. The estimated displacements in these areas

277 were precisely estimated due to the alluvial sediment background, which was adequately

278 coherent in both stacks. Points P3 and P4 exhibited faster subsidence rates than P2,

279 confirming the primary estimate from the differential interferograms of both stacks that these

280 regions were subsiding faster than the northern area.

281 On the other hand, the ascending stack could not provide precise estimates due to the

282 sparsity of acquisitions whereas local phase unwrapping failures in low coherent regions

283 were neither absent in the ascending pairs. Indicatively, the (multitemporal) coherence values

284 of points $\mathrm{P} 3$ and $\mathrm{P} 4$ were 0.48 and 0.26 respectively contrasted to 0.23 for both points in the

285 descending stack. Even though the time span of the acquisitions included two summer

286 periods, higher order models were not applied due to the extended low coherent regions and

287 the small number of scenes. Nevertheless, this short time interval was considered too short

288 to draw safe conclusions about the exact deformation behaviour of these areas.

289

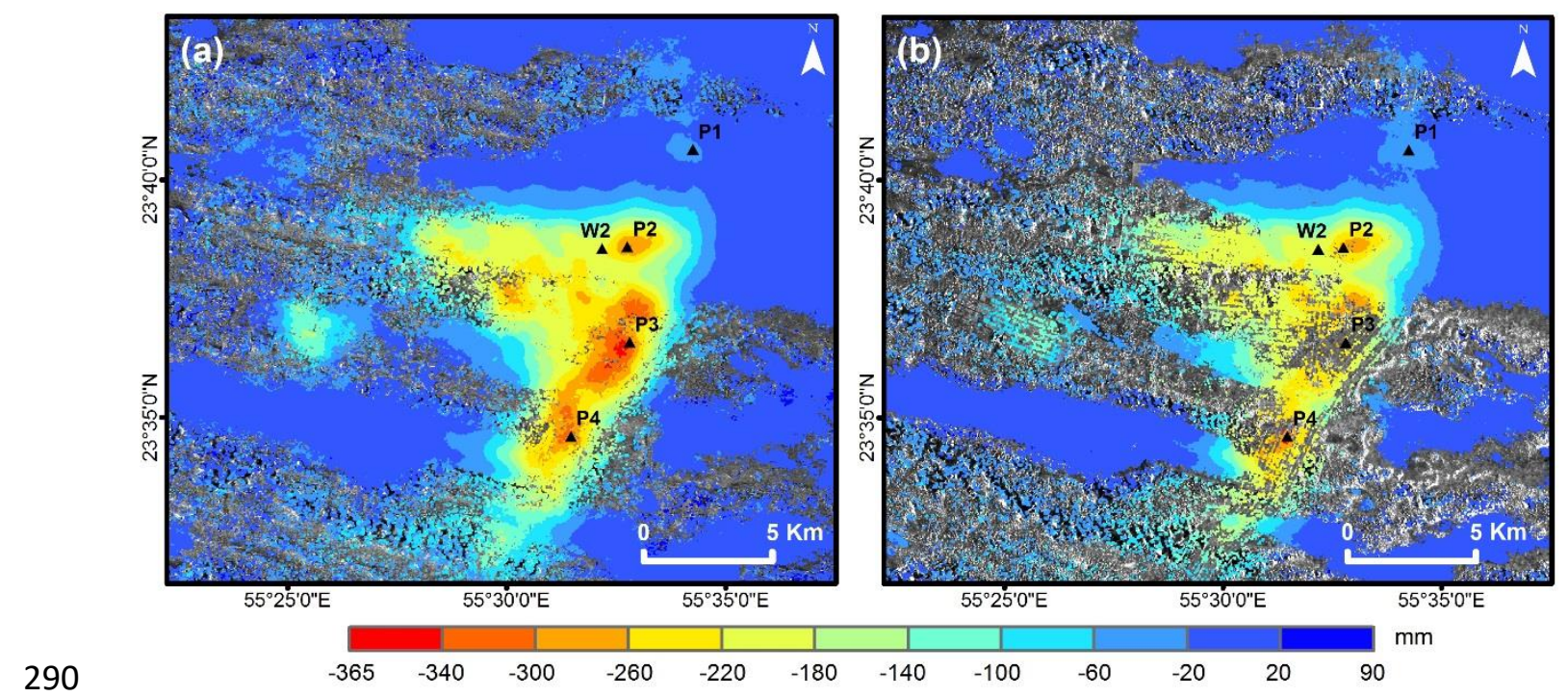



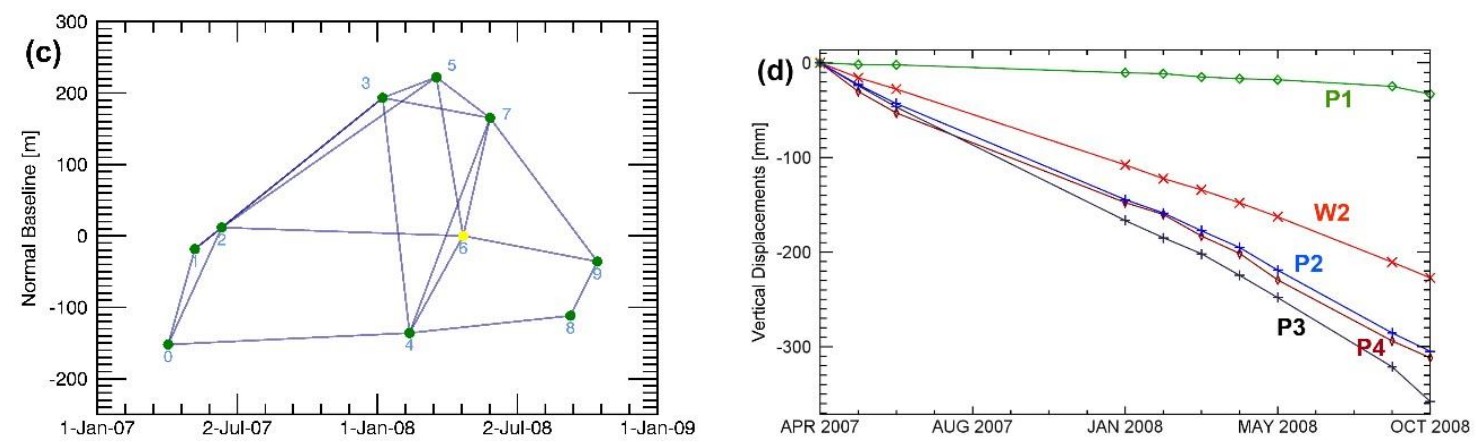

Figure 5. Comparison of the LOS displacements obtained from the two stacks with SBAS. a)

294 Ascending stack, 3/4/2007 - 14/10/2008. b) Descending stack, 29/3/2007 - 9/10/2008. c) Time 295 position plot of the descending stack images. d) Vertical displacement history derived from the 296 ascending stack (3/4/2007 - 14/10/2008).

297

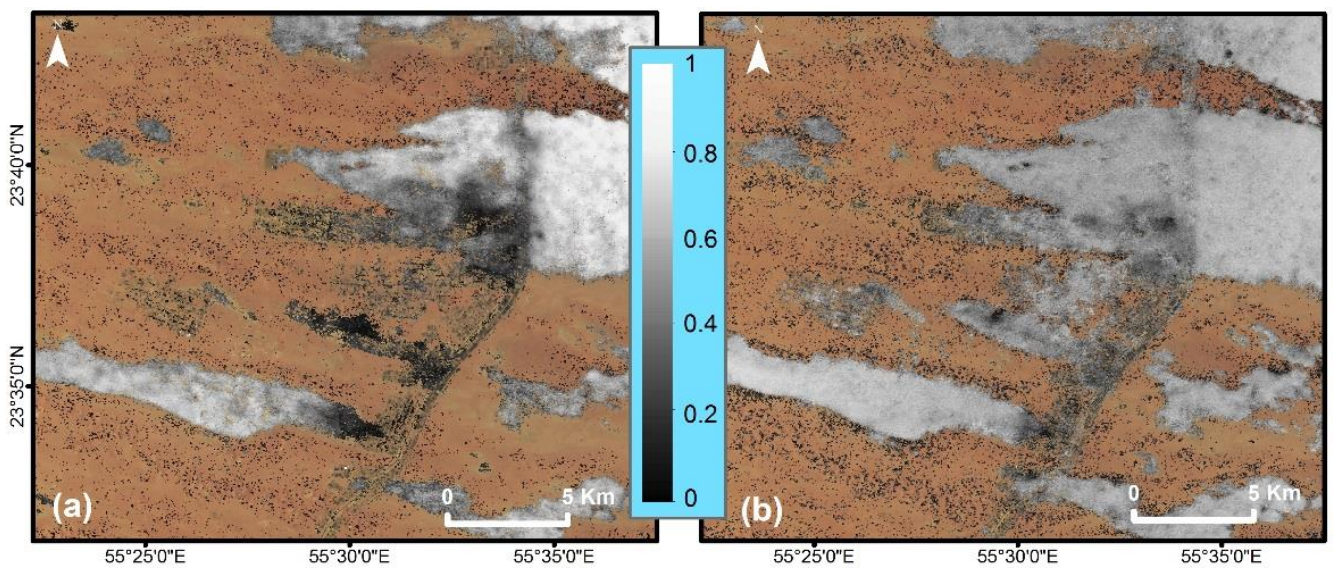

Figure 6. Multitemporal coherence. a) Descending stack. b) Ascending stack.

All possible interferograms within a temporal threshold of 700 days were calculated 304 from the $\mathrm{HH}$ polarization images of the PALSAR acquisitions as all normal baselines were 
305 less than $2300 \mathrm{~m}$. Interferograms between the FBD and FBS mode acquisitions were created 306 after oversampling the FBD images in range to the pixel spacing of the FBS scenes (Werner 307 et al., 2007). As it was expected, the L-band interferograms exhibited higher coherence levels 308 and greater spatial coverage providing with a more comprehensive picture of the deformation 309 pattern in longer time spans than the ENVISAT pairs. After the phase unwrapping stage, 16 310 interferograms were inverted with SBAS. The resulting deformation maps demonstrated an almost identical subsidence pattern

312 in terms of extent, even though non-systematic deviations were observed in comparison to 313 the C-band derived displacement maps that locally exceeded $2 \mathrm{~cm}$ when compared in similar 314 periods. Nonetheless, these differences were considered insignificant after taking into 315 account the small number of the scenes, the subsidence magnitude and the precision of 316 PALSAR in the achieved coherence levels (Pasquali et al., 2014). During the period 5/8/2007 $317-10 / 8 / 2009$, subsidence rates of $22 \mathrm{~cm} /$ year in the south, $21 \mathrm{~cm} /$ year in the central and 16 $318 \mathrm{~cm} /$ year in the north farmland areas were observed from the ALOS velocity map (figure 8a).

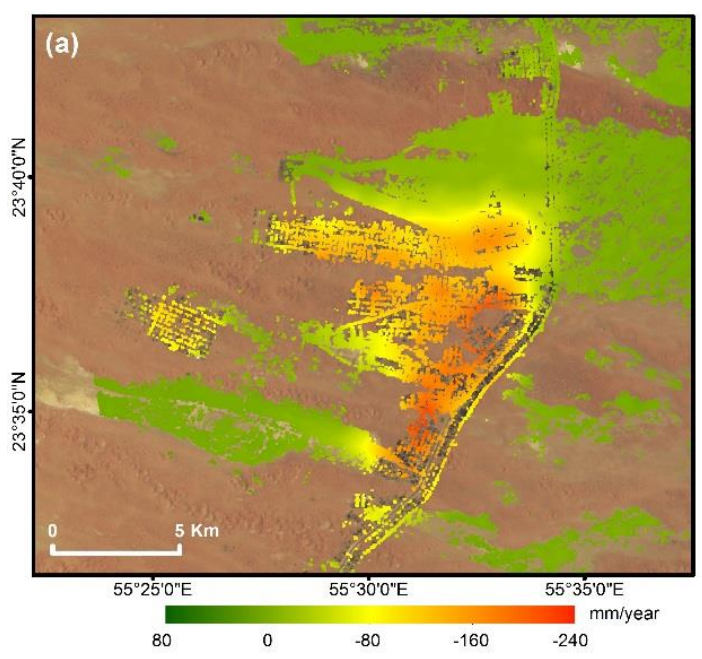




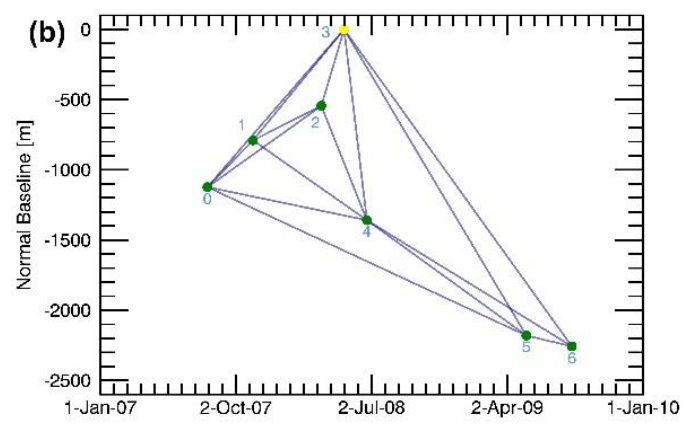

323 Figure 8. a) Subsidence rates obtained from the ALOS data. b) Time-baseline plot of the ALOS 324 images.

327 The Sentinel-1A images were multilooked $8 \times 2$ (range $\mathrm{x}$ azimuth) due to the lower fringe 328 rates and the differential interferograms (generated from the vertical polarization component) 329 verified the continuation of the subsidence phenomenon in a similar extent but with a lower magnitude. Deformation fringes are clearly visible in pairs that include the summer season

331 but they are not as prominent in the winter pair, indicating declining deformation trends that 332 might have become a seasonal phenomenon, even though this is still an assumption. The 333 generated wrapped interferograms are shown in figure 9. Results that provide stronger 334 evidence about the recent evolution of the terrain distortions will be obtained when the 335 number of acquisitions from Sentinel-1 will be sufficient for a time series interferometric 336 analysis and the atmospheric artefacts in the interferograms are compensated for. Similar 337 deformation trends were also observed in GPS data recordings of the Abu Dhabi GRS 338 Network station of Al Wagan (personal communication with Abu Dhabi Municipality, Town Planning sector, Spatial Data Division). 

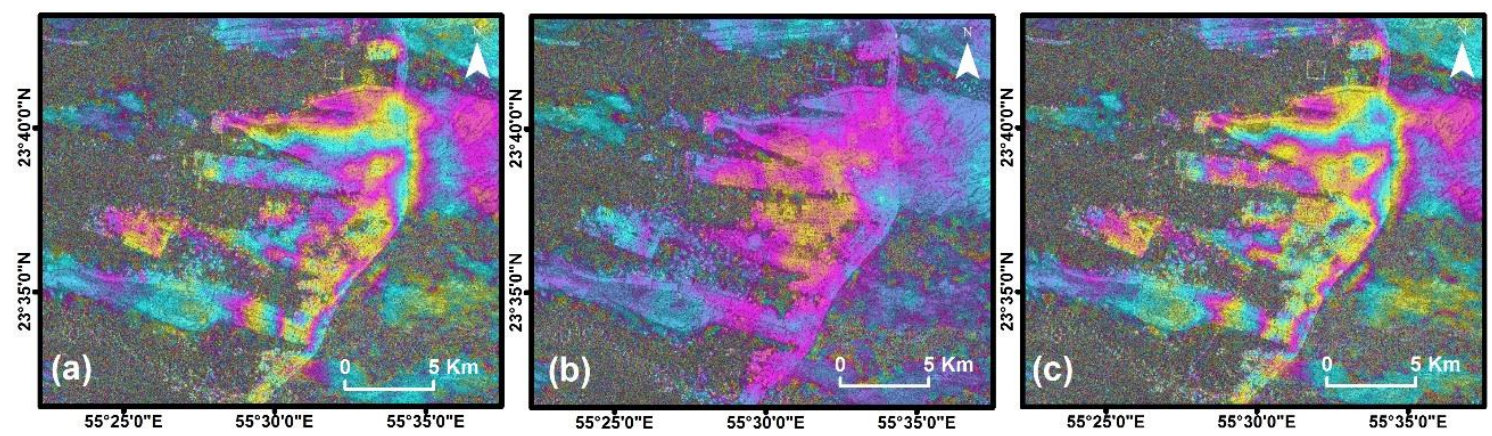

Figure 9. Differential interferograms from Sentinel-1A pairs. a) 18/2/2016 - 2/12/2016. b) 2/12/2016 - 8/3/2017. c) $18 / 2 / 2016-8 / 3 / 2017$.

\section{Correlation with groundwater levels}

346 Since the subsidence phenomena in Al Wagan were attributed to water pumping from the

347 subsurface, correlations with the groundwater levels were sought. We considered the water

348 level data from local wells monitored by the Environmental Agency of Abu Dhabi (EAD).

349 Even though these measurements were not concurrent and with the same frequency for all

350 the wells, their temporal patterns was examined to define whether a relationship of the 351 groundwater level fluctuations with the terrain deformations exists.

353 is inclined towards the west direction as mentioned in a previous study (Al Shahi, 2002). The

354 water level time series of the wells located within the north subsiding area are generally lower

355 contrasted to the surrounding region. This could be possibly related to groundwater extraction 356 which had started before the sampling period, but data prior to 2000 were not available. The 357 number of these wells is not adequate to extract conclusions about the spatial properties of 
358 the local aquifer as these should be examined in a wider area level with more similar datasets,

359 but an indication of these spatial characteristics is locally provided.

360 The main observation regards the declining trends exhibited from all of the 361 monitoring wells of the farmland area and the surroundings from 2000 and for the main 362 period of the DInSAR observations 2003-2010. In some of the wells, a recovery trend was 363 observed after 2012 without reaching the levels of 2000-2002, but in other cases the levels 364 were still reducing until September 2015. Inspection of Landsat 5 and 7 imagery from 1984 $365-2003$ showed that the land use scenery changed dramatically during the 1990s, as it was the 366 period of the farmland expansion. However, quantitative evidence about the start of the 367 aquifer resource exploitation was not available. Plots of the time series of the water levels 368 from selected representative wells are illustrated in figure 8.

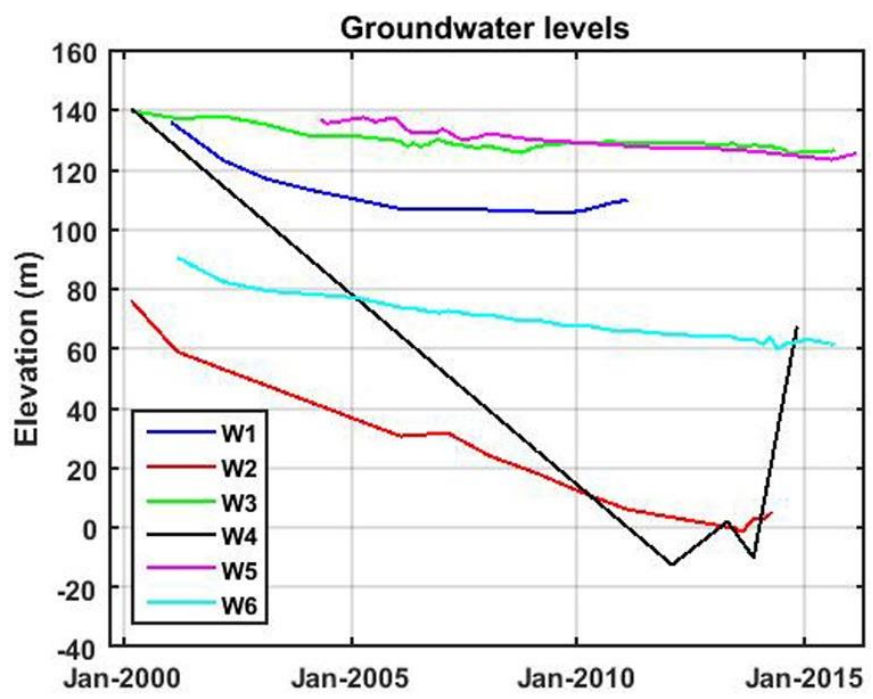




\section{Conclusions}

Interferometric processing over the agricultural area of Al Wagan revealed a localized subsidence phenomenon which is speculated to be groundwater related. It was observed from different sensors that subsidence velocities reached $20 \mathrm{~cm} /$ year during 2003-2010 with the terrain distortions exceeding $-1 \mathrm{~m}$. The land deformations of the study area would not have been detected if the resolution was lowered by large multilooking factors. Even though the area is not suitable for interferometric applications due to temporal decorrelation, DInSAR and SBAS techniques may still provide a terrain distortion metric if no other tools are available. The coherence maps were perfectly correlated with the land cover and the geological maps of the region, an aspect worthy of future investigation in order to define and develop the most appropriate techniques for mapping the surface deformations in the broader area of the UAE. The Sentinel-1A data verified that the surface deformation phenomena are still existent in the region constituting a potential hazard for the future.

According to the water level data, a correlation of the deformations with the groundwater resources exists. However, these data do not suffice to explain the seasonality of the subsidence pattern, while the fact that some areas are more affected than others even though the water level declinations appear similar or lower suggests that the local geology controls the phenomenon as well. The evaluation of the subsidence velocity field suggests that the loose sandy material background is more prone to terrain distortions than the compacted gravelly sediments of fluvial origin, but this also depends on the thickness of the formations. This aspect will be further sought in the geotechnical engineering properties of the different soil types of the study area by conducting in-situ sampling in the subsiding 
farmland area and correlating the different subsidence rates with both the aquifer resources 407 data. depletion and the geophysical parameters of the varying geological background.

Another aspect that could be correlated with the deformation pattern of the farmlands and the groundwater extraction for their maintenance regards the integration of optical imagery within this study. Since the agricultural areas kept expanding during the past 20 years and the land cover changes are captured in Landsat images, this type of changes should be reflected in the vegetation indices as well. It is expected that the time series analysis of these indices for this temporal interval will be capable of providing more comprehensive explanations about the excessive use of the aquifer resources in the area and the ground deformations that were induced by this process in the study area. Future work will also include accurate GPS measurements with Global Navigation Satellite Systems (GNSS) equipment to validate the deformation time series that will be derived from the Sentinel-1A

\section{References}

Al-Naeem, A. A. 2014. Effect of excess pumping on groundwater salinity and water level in Hail region of Saudi Arabia. Research Journal of Environmental Toxicology, 8, 124.

Al Shahi, F. A. 2002. Assessment of Groundwater Resources in Selected Areas of AI Ain in the UAE. Artesea, G., Fiaschib, S., Di Martirec, D., Tessitorec, S., Fabrisd, M., Achillid, V., Ahmedc, A., Borgstrome, S., Calcaterrac, D. \& Ramondinif, M. 2016. Monitoring of Land Subsidence in Ravenna Municipality Using Integrated SAR-GPS Techniques: Description and First Results. ISPRS-International Archives of the Photogrammetry, Remote Sensing and Spatial Information Sciences, 23-28. 
Bamler, R. \& Hartl, P. 1998. Synthetic aperture radar interferometry. Inverse problems, 14, R1.

Baran, I., Stewart, M. \& Claessens, S. 2005. A new functional model for determining minimum and maximum detectable deformation gradient resolved by satellite radar interferometry. IEEE Transactions on Geoscience and Remote Sensing, 43, 675-682.

Berardino, P., Fornaro, G., Lanari, R. \& Sansosti, E. 2002. A new algorithm for surface deformation monitoring based on small baseline differential SAR interferograms. IEEE Transactions on Geoscience and Remote Sensing, 40, 2375-2383.

Bürgmann, R., Rosen, P. A. \& Fielding, E. J. 2000. Synthetic aperture radar interferometry to measure Earth's surface topography and its deformation. Annual review of earth and planetary sciences, 28, 169-209.

Cantone, A., Riccardi, P., Baker, H., Pasquali, P., Closson, D. \& Karaki, N. A. Monitoring Small land Subsidence Phenomena in the Al-Ain Region by Satellite SAR Interferometric Stacking. Second EAGE International Conference on Engineering Geophysics, 2013.

Chaussard, E., Wdowinski, S., Cabral-Cano, E. \& Amelung, F. 2014. Land subsidence in central Mexico detected by ALOS InSAR time-series. Remote sensing of environment, 140, 94-106.

Environmental Agency of Abu Dhabi 2009. Abu Dhabi Water Resources Masterplan.

Ferretti, A. 2014. Satellite InSAR data: reservoir monitoring from space, EAGE Publications.

Ferretti, A., Prati, C. \& Rocca, F. 2000. Nonlinear subsidence rate estimation using permanent scatterers in differential SAR interferometry. IEEE Transactions on geoscience and remote sensing, 38, 2202-2212.

Ferretti, A., Prati, C. \& Rocca, F. 2001. Permanent scatterers in SAR interferometry. IEEE Transactions on geoscience and remote sensing, 39, 8-20.

Goldstein, R. M. \& Werner, C. L. 1998. Radar interferogram filtering for geophysical applications. Geophysical research letters, 25, 4035-4038. 
Gonzalez, R., Ouarda, T. B.M.J., Marpu, P. R., Allam, M. M., Eltahir, E. A. \& Pearson, S. 2016. Water Budget Analysis in Arid Regions, Application to the United Arab Emirates. Water, 8, 415.

Holzer, T. L. \& Galloway, D. L. 2005. Impacts of land subsidence caused by withdrawal of underground fluids in the United States. Reviews in Engineering Geology, 16, 87-99.

Hooper, A., Zebker, H., Segall, P. \& Kampes, B. 2004. A new method for measuring deformation on volcanoes and other natural terrains using InSAR persistent scatterers. Geophysical research letters, 31.

Kim, J.-W., Lu, Z., Jia, Y. \& Shum, C. 2015. Ground subsidence in Tucson, Arizona, monitored by timeseries analysis using multi-sensor InSAR datasets from 1993 to 2011. ISPRS Journal of Photogrammetry and Remote Sensing, 107, 126-141.

Lanari, R., Casu, F., Manzo, M., Zeni, G., Berardino, P., Manunta, M. \& Pepe, A. 2007. An overview of the small baseline subset algorithm: A DInSAR technique for surface deformation analysis. Pure and Applied Geophysics, 164, 637-661.

Lanari, R., Mora, O., Manunta, M., Mallorquí, J. J., Berardino, P. \& Sansosti, E. 2004. A small-baseline approach for investigating deformations on full-resolution differential SAR interferograms. IEEE Transactions on Geoscience and Remote Sensing, 42, 1377-1386.

Massonnet, D. \& Feigl, K. L. 1998. Radar interferometry and its application to changes in the Earth's surface. Reviews of geophysics, 36, 441-500.

Pasquali, P., Cantone, A., Riccardi, P., Defilippi, M., Ogushi, F., Gagliano, S. \& Tamura, M. 2014. Mapping of Ground Deformations with Interferometric Stacking Techniques. Land Applications of Radar Remote Sensing. InTech.

Pepe, A. \& Lanari, R. 2006. On the extension of the minimum cost flow algorithm for phase unwrapping of multitemporal differential SAR interferograms. IEEE Transactions on Geoscience and remote sensing, 44, 2374-2383. 
466 Statistics Centre of Abu Dhabi 2014. Agricultural land uses 2013 Statistical Report.

467 Strozzi, T., Wegmuller, U., Tosi, L., Bitelli, G. \& Spreckels, V. 2001. Land subsidence monitoring with 468 differential SAR interferometry. Photogrammetric engineering and remote sensing, 67, 469 1261-1270.

470 Vrba, J. 2003. The impact of aquifer intensive use on groundwater quality. Intensive use of 471 groundwater. Challenges and opportunities, 113-132.

472 Werner, C., Wegmüller, U., Strozzi, T., Wiesmann, A. \& Santoro, M. PALSAR multi-mode 473 interferometric processing. Proc. First Joint PI Symp. ALOS Data Nodes for ALOS Sci. $474 \quad$ Program, 2007. 19-23.

475 Yagoub, M. M. 2015. Spatio-temporal and hazard mapping of Earthquake in UAE (1984-2012): 476 Remote sensing and GIS application. Geoenvironmental Disasters, 2, 13. 